\title{
Computing the square roots of matrices with central symmetry*
}

\author{
Zhongyun Liu ${ }^{\dagger}$, Yulin Zhang ${ }^{\S}$ and Rui Ralha ${ }^{\ddagger}$
}

\begin{abstract}
For computing square roots of a nonsingular matrix $A$, which are functions of $A$, two well known fast and stable algorithms, which are based on the Schur decomposition of $A$, were proposed by Björk and Hammarling [3], for square roots of general complex matrices, and by Higham [10], for real square roots of real matrices. In this paper we further consider (the computation of) the square roots of matrices with central symmetry. We first investigate the structure of the square roots of these matrices and then develop several algorithms for computing the square roots. We show that our algorithms ensure significant savings in computational costs as compared to the use of standard algorithms for arbitrary matrices.
\end{abstract}

Keywords: Matrix square root, Schur algorithm, central symmetry

AMS Classifications: 65F30; $15 \mathrm{~A} 18$

\section{Introduction}

Theory and algorithms for structured matrices have been a topic of research for many years. There are many applications that generate structured matrices and by exploiting the structure one may be able to design faster and/or more accurate algorithms; furthermore, structure may also help in producing solutions which have more precise physical meaning. Structure comes in many forms, including Hamiltonian, Toeplitz, Vandermonde matrices and so on. Here, we consider the problem of the square roots of matrices with a central symmetric structure.

A matrix $X$ is said to be a square root of $A$ if $X^{2}=A$. The number of square roots varies from two (for a nonsingular Jordan block) to infinity (any involutary matrix is a square root of the identity matrix). If $A$ is singular, the existence of a square root depends on the Jordan structure of the zero eigenvalues. See for example [5] and Theorem 6.4.12 in [16]. If $A$ is real, it may or may not have a real square root, a sufficient condition for one to exist is that $A$ has no real negative eigenvalues, see for instance [10] and Theorem 6.4.14 in [16].

\footnotetext{
${ }^{*}$ Supported by: National Natural Science Foundation of China, No. 10571012; Postdoctoral Science Foundation of China; Excellent Youth Foundation of Educational Committee of Hunan Province, No. 04B006; Portuguese Foundation for Science and Technology (FCT) through the Research Programme POCTI.

${ }^{\dagger}$ Corresponding author: School of Mathematics and Computing Science, Changsha University of Science and Technology, Changsha, Hunan, 410076, P. R. China; Email: liuzhongyun@263.net

$\S^{\S}$ Department of Mathematics, University of Minho, Campus de Gualtar, 4710-057 Braga, Portugal; Email: zhang@math.uminho.pt

${ }^{\ddagger}$ Department of Mathematics, University of Minho, Campus de Gualtar, 4710-057 Braga, Portugal; Email: r_ ralha@math.uminho.pt
} 
The computational mehthods for square roots of a nonsingular matrices can be generally separated into two classes. The fisrt class of methods for computing the square root of a matrix are iterative methods. Matrix iterations $X_{j+1}=f\left(X_{j}\right)$, where $f$ is a polynomial or a rational function, are attractive alternatives to compute square roots for two reasons: they are readily implemented in terms of standard "building blocks", and they are potentially well suited to parallel computation. see for example, $[11,13,20]$ and reference therein. The second is the so-called direct method for computing square roots of a general complex matrix $A$ is the Schur algorithm developed by Björk and Hammarling for complex matrices [3]; Higham [10] has tailored the method for the real case. The method first computes the Schur decomposition $A=U T U^{H}$, where $U$ is unitary and $T$ is upper triangular; then finds $S$, upper triangular, such that $S^{2}=T$, using a fast recursion (viz., Parlett recurrence [22, 23]); finally, computes $X=U S U^{H}$, which is the desired square root of $A$. Any square root that is a function of $A$ can be computed by this approach. Furthermore, this method has been extended to compute matrix $p$ th roots [24] and general matrices functions [7].

As already said, our interest is focused in matrices with central symmetric structure. Recall that a matrix $A$ is said to be centrosymmetric if $A=\pi A \pi$, skew centrosymmetric if $A=-\pi A \pi$, centrohermitian if $A=\pi \bar{A} \pi$, where $\bar{A}$ denotes the elementwise conjugate of the matrix $A$ and $\pi$ is the exchange matrix with ones on the cross diagonal (bottom left to top right) and zeros elsewhere.

Symmetric (Hermitian) Toeplitz matrices form an important subclass of centrosymmetric (centrohermitian) matrices which naturally occur in digital signal processing and other areas, see, e.g., $[1,4,18]$. Centrosymmetric matrices appear in their own right, for example, in the numerical solution of certain differential equations [1], in the study of Markov processes [25] and in various physics and engineering problems [6]. Centrohermitian matrices arise in digital complex signal processing and image recovery, see for instance $[4,18]$.

This paper is organized as follows: after reviewing some basic properties of centrosymmetric (centrohermitian) matrices in next section, we investigate the structures of the square roots of centrosymmetric (centrohermitian) matrices in section 3 and develop some algorithms for computing those square roots in section 4. We end up with some conclusions and future work.

\section{Preliminaries}

Throughout this paper we denote the set of all $n \times n$ complex matrices by $\mathbb{C}^{n \times n}$ and the set of all $n \times n$ real matrices by $\mathbb{R}^{n \times n}$.

We first review the structure and reducibility of centrosymmetric (centrohermitian) matrices. All the formulas become slightly more complicated when $n$ is odd; for simplicity, we restrict our attention to the case of even $n=2 m$.

Using an appropriate partition, the central symmetric character of a $n \times n$ centrosymmetric matrix can be curved as follows:

$$
A=\left[\begin{array}{ll}
B & \pi_{m} C \pi_{m} \\
C & \pi_{m} B \pi_{m}
\end{array}\right]
$$


and the central symmetric character of a $n \times n$ skew centrosymmetric matrix can be always written as follows:

$$
A=\left[\begin{array}{ll}
B & -\pi_{m} C \pi_{m} \\
C & -\pi_{m} B \pi_{m}
\end{array}\right],
$$

where $B$ and $C$ are square matrices of order $m=n / 2$.

Defining

$$
P=\frac{\sqrt{2}}{2}\left[\begin{array}{cc}
I_{m} & I_{m} \\
\pi_{m} & -\pi_{m}
\end{array}\right],
$$

we have the following well known results, see, e.g., [1, 8, 19].

Lemma 1 (i). Let $P$ be defined as in (2.3). Then, $A$ is a $n \times n$ centrosymmetric matrix if and only if

$$
P^{T} A P=\left[\begin{array}{ll}
M & \\
& N
\end{array}\right]
$$

holds, where $N=B-\pi_{m} C$ and $M=B+\pi_{m} C$.

(ii). Let $P$ be defined as in (2.3). Then, $A$ is a $n \times n$ skew centrosymmetric matrix if and only if

$$
P^{T} A P=\left[\begin{array}{ll}
F^{E} &
\end{array}\right]
$$

holds, where $E=B+\pi_{m} C$ and $F=B-\pi_{m} C$.

Similarly, any $n \times n$ centrohermitian matrix always takes the following form:

$$
A=\left[\begin{array}{ll}
B & \pi_{m} \bar{C} \pi_{m} \\
C & \pi_{m} \bar{B} \pi_{m}
\end{array}\right] .
$$

Defining

$$
Q=\frac{\sqrt{2}}{2}\left[\begin{array}{cc}
I_{m} & i I_{m} \\
\pi_{m} & -i \pi_{m}
\end{array}\right],
$$

we have the following result, see for example $[8,14,17]$.

Lemma 2 Let $Q$ be defined as in (2.7). Then, $A$ is a $n \times n$ centrohermitian matrix if and only if

$$
Q^{H} A Q=R_{A}
$$

is a $n \times n$ real matrix, where

$$
R_{A}=\left[\begin{array}{cc}
\operatorname{Re}\left(B+\pi_{m} C\right) & -\operatorname{Im}\left(B+\pi_{m} C\right) \\
\operatorname{Im}\left(B-\pi_{m} C\right) & \operatorname{Re}\left(B-\pi_{m} C\right)
\end{array}\right],
$$

with $\operatorname{Re}(T)$ and $\operatorname{Im}(T)$ denoting the real and imaginary parts of the matrix $T$, respectively.

We will say that the matrix on the right side of $(2.4),(2.5)$ and $(2.8)$ is the reduced form of the matrix $A$ in (2.1), (2.2) and (2.6), respectively.

We end up this section with a well known result regarding the square roots of a nonsingular matrix [5] and a definition of matrix function [10, 24]. 
Lemma 3 [5, Lemma 1] Any nonsingular matrix always has a square root.

Definition 1 For a given function $f$ and a matrix $A \in \mathbb{C}^{n \times n}, f(A)$ can be defined as $g(A)$, where $g$ is a polynomial of minimal degree that interpolates $f$ on the spectrum of $A$, i.e.,

$$
g^{(j)}\left(\lambda_{k}\right)=f^{(j)}\left(\lambda_{k}\right), 0 \leq j \leq n_{k}-1,1 \leq k \leq s
$$

where $A$ has $s$ distinct eigenvalues $\lambda_{k}$ and $n_{k}$ is the largest Jordan block in which $\lambda_{k}$ appears.

In this paper we deal with the functions $f(\lambda)=\lambda^{\frac{1}{2}}$ and $f(\lambda)=\lambda^{\frac{1}{4}}$ which are clearly defined on the spectrum of a nonsingular matrix $A$.

\section{Square roots}

In this section we present some new results which characterize the square roots of nonsingular matrices with central symmetry.

\subsection{Square roots of centrosymmetric matrices}

It is known that the product of two centrosymmetric matrices is centrosymmetric and the product of two skew centrosymmetric matrices is also centrosymmetric. In fact, if $\pi B \pi=B$, or $\pi B \pi=-B$, then $A=B^{2}$ implies $\pi A \pi=A$. One may ask whether a centrosymmetric matrix has square roots which are also centrosymmetric or skew centrosymmetric. We have some answers to this question.

Theorem 1 Let $A \in \mathbb{C}^{n \times n}$ be centrosymmetric. Then, $A$ has a centrosymmetric square root if and only if each of $M$ and $N$ in (2.4) admits a square root.

Proof. From the hypothesis, we have that $A$ is centrosymmetric. Then, by Lemma 1, A has the reduced form (2.4).

$\Longrightarrow$. Assume that $A$ has a centrosymmetric square root, denoted by $\tilde{X}$. Again, using Lemma 1, we have

$$
P^{T} \tilde{X} P=X=\left[\begin{array}{ll}
X_{1} & \\
& X_{2}
\end{array}\right],
$$

where $P$ is defined as in (2.3).

Note that $\tilde{X}^{2}=A$ implies that $X_{1}^{2}=M$ and $X_{2}^{2}=N$ hold simultaneously, i.e., $X_{1}$ and $X_{2}$ are square roots of $M$ and $N$, respectively.

$\Longleftarrow$. If $M$ and $N$ in (2.4) have square roots $X_{1}$ and $X_{2}$, respectively, then $X=\left[\begin{array}{ll}X_{1} & \\ & X_{2}\end{array}\right]$ is a square root of the matrix $\left[\begin{array}{cc}M & \\ & N\end{array}\right]$. By Lemma 1, $\tilde{X}=P X P^{T}$ is a centrosymmetric square root of $A$. This means that $A$ always has a centrosymmetric square $\operatorname{root} \tilde{X}$. 
Corollary $1 A$ nonsingular centrosymmetric matrix $A \in \mathbb{C}^{n \times n}$ always has a centrosymmetric square root.

Proof. Because $A$ is centrosymmetric and nonsingular, the matrices $M$ and $N$ in the reduced form (2.4) of $A$ are nonsingular. Then, by Lemma 3, each of $M$ and $N$ has a square root. Therefore, by Theorem 1, we know that $A$ always has a centrosymmetric square root.

It was shown in [20] that the principal square root of a centrosymmetric matrix having no eigenvalues on the nonpositive real axis is centrosymmetric. We now present another result.

Theorem 2 If $A \in \mathbb{C}^{n \times n}$ is a nonsingular centrosymmetric matrix, then all square roots of $A$ which are functions of $A$ are centrosymmetric.

Proof. Assume that $\tilde{X}$ is a square root of $A$ which is a function of $A$, that is, $\tilde{X}^{2}=A$ and $\tilde{X}=f(A)$. Using the fact that the sum and product of two centrosymmetric matrices are also centrosymmetric, and $f(A)=g(A)$ with $g(A)$ a polynomial in $A$, we then have that $\tilde{X}=g(A)$ is obviously centrosymmetric.

Remark 1 Assume that $\tilde{X}$ is a square root of $A$ which is a function of $A$. Then, by Lemma 1 and Theorem 2, we have that

$$
P^{T} \tilde{X} P=X=\left[\begin{array}{ll}
X_{1} & \\
& X_{2}
\end{array}\right] \text { and } P^{T} A P=\left[\begin{array}{ll}
M & \\
& N
\end{array}\right],
$$

which means that $X_{1}=f(M)$ and $X_{2}=f(N)$. Thus, the problem of computing square roots of a centrosymmetric matrix $A$, which are functions of $A$, can be reduced to that of computing square roots of two half size matrices $M$ and $N$, which are functions of $M$ and $N$, respectively. This is the basis of Algorithm 1 that will be given in section 4 .

We have shown that a nonsingular centrosymmetric matrix always has a centrosymmetric square root. However, it is not true that every centrosymmetric matrix has a skew centrosymmetric square root.

Lemma 4 A nonsingular centrosymmetric matrix with odd order has no skew centrosymmetric square roots.

Proof. By lemma 3, we know that $A$ has a square root, which we denote by $Y$. Assume that $Y$ is skew centrosymmetric, viz. $\pi Y \pi=-Y$ and $Y^{2}=A$. We then have that $\operatorname{det}(A)=(\operatorname{det} Y)^{2}$ and $\operatorname{det}(Y)=(-1)^{n} \operatorname{det}(Y)$. Since $n$ is odd, we get $\operatorname{det}(Y)=0$ and $\operatorname{det}(A)=0$. This leads to a contradiction with the assumption of nonsingularity of $A$. Therefore $A$ has no skew centrosymmetric square roots.

\footnotetext{
"Any matrix $A$ having no nonpositive real eigenvalues has a unique square root for which every eigenvalue has positive real part, denoted by $A^{1 / 2}$ and called the principal square root; That is, $\left(A^{\frac{1}{2}}\right)^{2}=A$ and $\operatorname{Re}\left(\lambda_{k}\right)>0$ for all $k$, where $\lambda_{k}(A)$ denotes an eigenvalue of $A$.
} 
Theorem 3 Let $A \in \mathbb{C}^{n \times n}$ be a nonsingular centrosymmetric matrix. Then, $A$ has a skew centrosymmetric square root if and only if $M$ and $N$ in (2.4) are similar. Furthermore, if $A$ has a skew centrosymmetric square root, then $A$ has infinitely many skew centrosymmetric square roots.

Proof. $\Longrightarrow$. Since $A$ is a nonsingular centrosymmetric matrix, by Lemma 1, we have that $M$ and $N$ in (2.4) are nonsingular. If $A$ has a skew centrosymmetric square root $\tilde{Y}$, then by Lemma 1 , we have $P^{T} \tilde{Y} P=\left[\begin{array}{cc}Y_{1} \\ Y_{2}\end{array}\right]$. Also, by Lemma 4, we have that $n$ must be even. This means that $M, N, Y_{1}$ and $Y_{2}$ are of the same order $n / 2$.

Note that $\tilde{Y}^{2}=A$ means that $Y_{1} Y_{2}=M$ and $Y_{2} Y_{1}=N$. Therefore, $Y_{1}$ and $Y_{2}$ are both nonsingular (due to the non-singularity of $M$ and $N$ ) and $N=Y_{1}^{-1} M Y_{1}=Y_{2} M Y_{2}^{-1}$.

$\Longleftarrow$. If $M$ and $N$ are similar, then there exists a nonsingular matrix $S$ such that $S^{-1} M S=N$, i.e., $M S=S N$. Setting $Y_{1}=S$ and $Y_{2}=S^{-1} M$ gives $Y_{2} Y_{1}=N$ and $Y_{1} Y_{2}=M$. In this situation, $Y=\left[\begin{array}{cc} & Y_{1} \\ Y_{2} & \end{array}\right]$ is a square root of $\operatorname{diag}(M, N)$. Thus, by Lemma 1 again, $\tilde{Y}=P Y P^{T}$ is a skew centrosymmetric square root of $A$.

Finally, to prove that if $A$ has a skew centrosymmetric square root, then $A$ has infinitely many skew centrosymmetric square roots, it suffices to take $S=k Y_{1}$, with $k$ an arbitrary nonzero constant, and use the preceding reasoning.

In general, a nonsingular centrosymmetric matrix $A$, besides the centrosymmetric square roots, possibly has other kinds of square roots (for example skew centrosymmetric square roots), which are not functions of $A$. The existence and the families of square roots depend on the spectrums of $M$ and $N$. The following theorem gives a classification of all the square roots of a nonsingular centrosymmetric matrix.

Theorem 4 Let $A \in \mathbb{C}^{n \times n}$ be nonsingular and centrosymmetric with reduced form given by (2.4). Assume that $M$ has $s$ distinct eigenvalues and $N$ has $t$ distinct eigenvalues. Let $M=Z_{M} J_{M} Z_{M}^{-1}$ with $J_{M}=\operatorname{diag}\left(J_{1}, J_{2}, \ldots, J_{l}\right)$ and $N=Z_{N} J_{N} Z_{N}^{-1}$ with $J_{N}=\operatorname{diag}\left(\breve{J}_{1}, \check{J}_{2}, \ldots, \breve{J}_{r}\right)$ be the Jordan decompositions of $M$ and $N$ respectively.

i) If $\sigma(M) \bigcap \sigma(N)=\phi$, where $\sigma(W)$ denotes the spectrum of matrix $W$, then $A$ has $2^{s+t}$ centrosymmetric square roots which are functions of $A$ and take the form

$$
\hat{X}=P Z \hat{L} Z^{-1} P^{T}
$$

with

$$
Z=\operatorname{diag}\left(Z_{M}, Z_{N}\right), \quad \hat{L}=\operatorname{diag}\left(\hat{L}_{M}, \hat{L}_{N}\right)
$$

where $\hat{L}_{M}$ denotes a square root of $J_{M}$, which is a function of $J_{M}$ and $\hat{L}_{N}$ denotes a square root of $J_{N}$, which is a function of $J_{N}$. Furthemore, if $s+t<$ $l+r$ then $A$ has centrosymmetric square roots which are not functions of $A$ and 
they form $2^{l+r}-2^{s+t}$ parameterized families given by

$$
\tilde{X}=P Z \operatorname{diag}\left(U_{M}, U_{N}\right) \hat{L} \operatorname{diag}\left(U_{M}^{-1}, U_{N}^{-1}\right) Z^{-1} P^{T}
$$

where $U_{M}$ and $U_{N}$ are two arbitrary nonsingular matrices which commute with $J_{M}$ and $J_{N}$, respectively.

ii) If $M$ and $N$ have $\alpha$ common eigenvalues, then $A$ has $2^{s+t-\alpha}$ centrosymmetric square roots which are functions of $A$, taking the form given in (3.1); and $A$ has centrosymmetric square roots which are not functions of $A$; they form $2^{l+r}-2^{s+t}$ parameterized families taking the form given in (3.3). Furthermore, $A$ has square roots which are not centrosymmetric and are not functions of $A$, they form $2^{s+t}-2^{s+t-\alpha}$ parameterized families taking the following form:

$$
\tilde{X}(U)=P Z U \hat{L} U^{-1} Z^{-1} P^{T},
$$

where $U$ is an arbitrary nonsingular matrix which commutes with $J=\operatorname{diag}\left(J_{M}\right.$, $\left.J_{N}\right)$ but does not have the form such as $\operatorname{diag}\left(U_{M}, U_{N}\right)$.

Proof. i) We just need to apply Theorem 2 and Theorem 4 in [10] to $\operatorname{diag}(M, N)$ which, according to the hypothesis, has $s+t$ distinct eigenvalues and $l+r$ Jordan blocks. The centrosymmetry of the square roots in (3.1), which are functions of $A$, is guaranteed by Theorem 2. For the square roots of the form (3.3), which are not functions of $A$, we note that $\hat{L}$ is a $2 \times 2$ block diagonal matrix and $Z \hat{L} Z^{-1}$ and $Z \operatorname{diag}\left(U_{M}, U_{N}\right) \hat{L} \operatorname{diag}\left(U_{M}, U_{N}\right)^{-1} Z^{-1}$ are also $2 \times 2$ block diagonal matrices.

By Lemma 1, we conclude that these matrices are also centrosymmetric.

ii) If $M$ and $N$ have $\alpha$ common eigenvalues, then $A$ has $s+t-\alpha$ distinct eigenvalues. By Theorem 4 in [10] again, we then have that $A$ has $2^{s+t-\alpha}$ square roots which are functions of $A$ and take the form (3.1). By Theorem 2, those square roots are centrosymmetric. Furthermore, $A$ has square roots which are not functions of $A$, they form $2^{l+r}-2^{s+t-\alpha}$ parameterized families, which can be expressed as the following form

$$
\check{X}(V)=P Z V \hat{L} V^{-1} Z^{-1} P^{T},
$$

where $V$ is an arbitrary nonsingular matrix which commutes with $J$.

Those square roots consist of two groups: those that are centrosymmetric and those that are not centrosymmetric. By Theorem 1 and Lemma 1 as well as the first part, those square roots which are centrosymmetric take the form as in (3.3), they form $2^{l+r}-2^{s+t}$ parameterized families.

The remaining square roots are not centrosymmetric or functions of $A$, they form $2^{s+t}-2^{s+t-\alpha}$ parameterized families and can be written as the following form:

$$
\tilde{X}(U)=P Z U \hat{L} U^{-1} Z^{-1} P^{T},
$$

where $U$ is not able to be written as the form $\operatorname{diag}\left(U_{M}, U_{N}\right)$. Thus we complete the proof. 


\subsection{Square roots of skew centrosymmetric matrices}

If $A$ is a $n \times n$ nonsingular skew centrosymmetric matrix, then Lemma 4 implies that $n$ is even. Let $(\lambda, x)$ be an eigenpair of $A$. From $\pi A \pi=-A$ we get $A \pi x=-\lambda \pi x$ which means that the eigenvalues of $A$ must appear in \pm pairs, and $A$ has a Jordan decomposition of the form

$$
A=S \operatorname{diag}(J, \hat{J}) S^{-1}
$$

with

$$
J=\operatorname{diag}\left(J_{1}, J_{2}, \ldots, J_{l}\right), \quad \hat{J}=\operatorname{diag}\left(\hat{J}_{1}, \hat{J}_{2}, \ldots, \hat{J}_{l}\right)
$$

where

$$
J_{j}=\lambda_{j} I+E, \quad \hat{J}_{j}=-\lambda_{j} I+E, \quad E=\left(\delta_{p, q-1}\right)
$$

are $m_{j} \times m_{j}$ matrices such that $\sum_{j=1}^{l} m_{j}=n / 2$.

Assume that $J$ has $s$ distinct eigenvalues. We have the following result.

Theorem 5 Let the nonsingular skew centrosymmetric matrix $A \in \mathbb{C}^{n \times n}$, of order even, have the Jordan decomposition (3.6), and let $s \leq l$ be the number of distinct eigenvalues of $J$. Then $A$ has $4^{s}$ square roots, which are functions of $A$, taking the form

$$
X=S \operatorname{diag}(\hat{L}, L) S^{-1},
$$

where $\hat{L}$ is a square root of $J$ and $L$ is a square root of $\hat{J}$.

If $s<l$, then $A$ has square roots which are not functions of $A$, they form $4^{l}-4^{s}$ parameterized families, which can be written as

$$
X(U)=S U \operatorname{diag}(\hat{L}, L) U^{-1} S^{-1},
$$

where $U$ is an arbitrary nonsingular matrix which commutes with $\operatorname{diag}(J, \hat{J})$.

Proof. The proof consists in using again Theorem 4 in [10] and the fact that $A$ has $2 s$ distinct eigenvalues and $2 l$ Jordan blocks.

From the computational point of view, we now consider the structure of the square roots of a nonsingular skew centrosymmetric matrix $A$, which are functions of $A$. Let $A$ be partitioned as in (2.2). By Lemma 1, we have that $P^{T} A P$ takes the form (2.5). Exploiting this form, we have the following theorem:

Theorem 6 Let $A \in \mathbb{C}^{n \times n}$ be a nonsingular skew centrosymmetric matrix of even order $n$. Assume that two matrices $\tilde{Z}, Z \in \mathbb{C}^{n \times n}$, with $\tilde{Z}=P Z P^{T}$, are partitioned as follows

$$
Z=\left[\begin{array}{ll}
Z_{1} & Z_{2} \\
Z_{3} & Z_{4}
\end{array}\right], \quad \tilde{Z}=\left[\begin{array}{ll}
\tilde{Z}_{1} & \tilde{Z}_{2} \\
\tilde{Z}_{3} & \tilde{Z}_{4}
\end{array}\right]
$$

which are conformable with the partition of $A$ in (2.2). Then $\tilde{Z}$ is a square root of $A$ if and only if

(A) $Z_{2} Z_{3}$ is a square root of $-\frac{1}{4} E F$ 
(B) $Z_{1}$ is a fourth root of $-\frac{1}{4} E F$;

(C) $Z_{4}$ is a fourth root of $-\frac{1}{4} F E$;

(D) $Z_{2}$ is a solution of $Z_{1} Z_{2}+Z_{2} Z_{4}=E$;

(E) $Z_{3}=E^{-1} Z_{2} F$;

hold simultaneously, where $E, F$ and $P$ are defined as in (2.5) and (2.3), respectively.

Proof. If $A$ is nonsingular and skew centrosymmetric, then $A$ has a square root. Furthermore, we have that $E$ and $F$ in (2.5) are two nonsingular matrices of order $\frac{n}{2}$.

$\Longrightarrow$. Let $\tilde{Z}$ denote a square root of $A$, i.e., $\tilde{Z}^{2}=A$. Then $P^{T} \tilde{Z}^{2} P=$ $\left(P^{T} \tilde{Z} P\right)^{2}=Z^{2}=P^{T} A P$ gives

$$
\left[\begin{array}{ll}
Z_{1} & Z_{2} \\
Z_{3} & Z_{4}
\end{array}\right]^{2}=\left[\begin{array}{ll} 
& E \\
F &
\end{array}\right]
$$

subject to

$$
\left[\begin{array}{ll}
Z_{1} & Z_{2} \\
Z_{3} & Z_{4}
\end{array}\right]\left[\begin{array}{ll} 
& E \\
F &
\end{array}\right]=\left[\begin{array}{ll} 
& E \\
F &
\end{array}\right]\left[\begin{array}{ll}
Z_{1} & Z_{2} \\
Z_{3} & Z_{4}
\end{array}\right] \|
$$

holds.

A straightforward expansion of (3.8) gives

$$
\left\{\begin{array} { l } 
{ Z _ { 1 } ^ { 2 } + Z _ { 2 } Z _ { 3 } = 0 } \\
{ Z _ { 1 } Z _ { 2 } + Z _ { 2 } Z _ { 4 } = E } \\
{ Z _ { 3 } Z _ { 1 } + Z _ { 4 } Z _ { 3 } = F } \\
{ Z _ { 4 } ^ { 2 } + Z _ { 3 } Z _ { 2 } = 0 }
\end{array} \quad ( \text { subject to } ) \quad \left\{\begin{array}{l}
E Z_{3}=Z_{2} F \\
E Z_{4}=Z_{1} E \\
F Z_{1}=Z_{4} F \\
F Z_{2}=Z_{3} E
\end{array}\right.\right.
$$

Assume that $Z_{2}$ is nonsingular. It follows that $Z_{1}, Z_{3}$ and $Z_{4}$ are also nonsingular. $^{* *}$ Therefore $Z_{2} Z_{3}$ being nonsingular means that $-Z_{2} Z_{3}$ has a square root, say $X$. Also, because $-Z_{3} Z_{2}$ is similar to $-Z_{2} Z_{3}, Y=Z_{3} X Z_{3}^{-1}$ is a square root of $-Z_{3} Z_{2}$.

Due to $Z_{1} Z_{2}+Z_{2} Z_{4}=E$, we have $X Z_{2} Z_{3}+Z_{2} Z_{3} X=E Z_{3}$, which implies that $X^{3}=-\frac{1}{2} E Z_{3}$. From (3.9), we know that $E Z_{3}=Z_{2} F$ and we can write $X^{6}=\left(-Z_{2} Z_{3}\right)^{3}=\frac{1}{4} Z_{2} F E Z_{3}$, which gives $-\left(Z_{2} Z_{3}\right)^{2} Z_{2}=\frac{1}{4} Z_{2} F E$. Since $\left(Z_{2} Z_{3}\right)^{2} Z_{2}=Z_{2}\left(Z_{3} Z_{2}\right)^{2}$, we get that $\left(Z_{3} Z_{2}\right)^{2}=-\frac{1}{4} F E$. Moreover, using $E Z_{3}=Z_{2} F$ and $F Z_{2}=Z_{3} E$, we obtain that $\left(Z_{2} Z_{3}\right)^{2}=Z_{2}\left(Z_{3} Z_{2}\right)^{2} Z_{2}^{-1}=$ $-\frac{1}{4} Z_{2} F E Z_{2}^{-1}=-\frac{1}{4} E F$. Also, since $X^{2}=-Z_{2} Z_{3}$ and $Y^{2}=-Z_{3} Z_{2}$, we have that $X$ is a fourth root of $-\frac{1}{4} E F$, and $Y$ is a fourth root of $-\frac{1}{4} F E$.

By now, we have proved that if $\tilde{Z}$ is a square root of $A$ and if $Z_{2}$ is nonsingular, then statements (A), (B), (C), (D) and (E) hold. The remaining is to show that $Z_{2}$ must be nonsingular. Note that

$$
Z_{1} Z_{2}+Z_{2} Z_{4}=E
$$

\footnotetext{
"If $X$ is a square root of $A$, then $A X=X A$.

** In general, if we assume that one of the matrices $\left\{Z_{j}\right\}_{j=1}^{4}$ in (3.9) is nonsingular, then it follows that the other 3 matrices are also nonsingular.
} 
can be written as

$$
\left[\begin{array}{ll}
Z_{1} & Z_{2}
\end{array}\right]\left[\begin{array}{l}
Z_{2} \\
Z_{4}
\end{array}\right]=E
$$

Because $\mathcal{N}(A)=\mathcal{N}\left(A^{2}\right)^{\dagger \dagger}$ and $\mathcal{N}(A B) \supseteq \mathcal{N}(B)$ for any matrices $A$ and $B$, of order $n$ (see, for example, [21, Ex. 4.5.18, (a)], and [21, Ex. 4.2.12, (b)]), from (3.9) we have that

$$
\mathcal{N}\left(Z_{4}\right)=\mathcal{N}\left(Z_{4}^{2}\right)=\mathcal{N}\left(Z_{3} Z_{2}\right) \supseteq \mathcal{N}\left(Z_{2}\right) .
$$

If $Z_{2}$ is singular, then $\mathcal{N}\left(Z_{2}\right)$ is nontrivial. Let $x \neq 0 \in \mathcal{N}\left(Z_{2}\right) \subseteq \mathcal{N}\left(Z_{4}\right)$. We have that

$$
E x=\left[\begin{array}{ll}
Z_{1} & Z_{2}
\end{array}\right]\left[\begin{array}{l}
Z_{2} \\
Z_{4}
\end{array}\right] x=0,
$$

which implies that $\mathcal{N}(E)$ is nontrivial. This yields a contradiction with the nonsingularity of $E$. So $Z_{2}$ must be nonsingular.

$\Longleftarrow$. Assume that (A), (B), (C), (D) and (E) hold. Since $A$ is nonsingular, $E$ and $F$ are also nonsingular. Then, from (E), i.e., $Z_{3}=E^{-1} Z_{2} F$, we have $E Z_{3}=$ $Z_{2} F$. Combined with (D), i.e., $Z_{1} Z_{2}+Z_{2} Z_{4}=E$, we have $Z_{3} Z_{1}+Z_{4} Z_{3}=F$. Denoting by $X$ a fourth root of $-\frac{1}{4} E F$ which corresponds to the square root of $-Z_{2} Z_{3}$ and by $Y$ a fourth root of $-\frac{1}{4} F E$ which corresponds to the square root of $-Z_{3} Z_{2}$ and forming $Z=\left[\begin{array}{cc}X & Z_{2} \\ Z_{3} & Y\end{array}\right]$ show that $Z^{2}=\left[\begin{array}{cc} & E \\ F & \end{array}\right]$. That is to say that $\tilde{Z}=P Z P^{T}$ is a square root of $A$.

We give a remark to end this subsection.

Remark 2 1. Unlike nonsingular centrosymmetric matrices, which always have centrosymmetric square roots, a nonsingular skew centrosymmetric matrix A has no specially structured square roots, see Theorem 6.

2. From the computational point of view, Theorem 6 provides an efficient approach to computing the square roots of a nonsingular skew centrosymmetric matrix A, see Algorithm 2. In this algorithm, we need to compute a fourth root of $-\frac{1}{4} E F$, which is a function of $-\frac{1}{4} E F$ (for more details concerning pth roots, see [24]), and solve a Sylvester equation $X Z_{2}+Z_{2} Y=E$ which has a unique solution for a given $X$ having no repeated eigenvalues, because $X$ and $Y$ are similar. By Theorem 2.2 in [24] we know that a nonsingular matrix $B \in \mathbb{C}^{n \times n}$ with $s$ distinct eigenvalues has precisely $4^{s}$ fourth roots which are function of $B$. Furthermore, since $A$ is skew centrosymmetric, $A$ has $n / 2$ distinct \pm eigenvalues, which implies that $E F$ has $n / 2$ distinct eigenvalues. So $\frac{1}{4} E F$ has $4^{\frac{n}{2}}=2^{n}$ fourth roots which are functions of $\frac{1}{4} E F$. In this situation, equation $X Z_{2}+Z_{2} Y=E$ has precisely $2^{n}$ solutions. This means we can form $2^{n}$ square roots of $A$ which are functions of $A$, this coincides with the conclusion of Theorem 5 .

\footnotetext{
${ }^{\dagger \dagger} \mathcal{N}(A)$ denotes the nullspace of the matrix $A$.
} 


\subsection{Square roots of centrohermitian matrices}

In this subsection we look at the square roots of a nonsingular centrohermitian matrix $A$. Due to $\pi A \pi=\bar{A}$ we have that $B=A^{2}$ is also centrohermitian. However, we find that not every centrohermitian matrix has a centrohermitian square root.

Theorem 7 A nonsingular centrohermitian matrix $A \in \mathbb{C}^{n \times n}$ has a centrohermitian square root if and only if $A$ 's reduced form $R_{A} \in \mathbb{R}^{n \times n}$ in (2.8) has a real square root.

Proof. $\Longrightarrow$. Assume that $A$ is a centrohermitian matrix and has a centrohermitian square root $Y$, i.e., $Y^{2}=A$. Then, by Lemma 2 , we have that $R_{Y}=Q^{H} Y Q$ and $R_{A}=Q^{H} A Q$ are real and $R_{Y}^{2}=R_{A}$, where $Q$ is defined in (2.7). This means that $R_{A}$ has a real square root.

$\Longleftarrow$. If $R_{A}$ has a real square root $C$, i.e., $R_{A}=C^{2}$. By Lemma 2, we then have that $A=Q R_{A} Q^{H}$ and $Y=Q C Q^{H}$ are centrohermitian. Furthermore, we have that $A=Y^{2}$, which means that $Y$ is a centrohermitian square root.

It is showed in Theorem 2 that all the square roots of a nonsingular centrosymmetric matrix $A$ which are functions of $A$ are centrosymmetric. But for nonsingular centrohermitian matrices, this conclusion does not hold anymore in general. However, if a square root of a nonsingular centrohermitian matrix $A$ is a real coefficient polynomial in $A$, then this conclusion does hold.

Corollary 2 If $A \in \mathbb{C}^{n \times n}$ is nonsingular and centrohermitian, then all square roots of $A$ which are polynomials in $A$ with real coefficients (if exist) are centrohermitian.

Proof. Assume that $Y$ is a square root of $A$ which is a polynomial in $A$ with real coefficients; that is, $Y^{2}=A$ and $Y=g(A)$, where all coefficients of $g(A)$ are real. Using the fact that the sum and product of two centrihermitian matrices are also centrohermitian, we then have that $Y=g(A)$ is centrohermitian.

Clearly, whether $A$ has a centrohermitian square root or not is equivalent to whether $R_{A}$ has a real square root or not.

Corollary 3 Let $A \in \mathbb{C}^{n \times n}$ be nonsingular and centrohermitian. $A$ has a centrohermitian square root if and only if each elementary divisor of $A$ corresponding to a real negative eigenvalue occurs an even number of times.

Proof. The proof is a straightforward extension of Theorem 5 in [10] by using the fact $|\lambda I-A|=\left|\lambda I-R_{A}\right|$, and is omitted.

At last, we give a classification of the square roots of a nonsingular centrohermitian matrix $A$. Assume that $A$ is a nonsingular centrohermitian matrix, $\lambda$ is an eigenvalue of $A$ and $x$ is a eigenvector corresponding to $\lambda$, viz., $A x=\lambda x$. Because $\pi A \pi=\bar{A}$, we have that $A \pi x=\bar{\lambda} \pi x$, which means the complex eigenvalues of $A$ must appear in conjugate pairs, and $A$ has a Jordan decomposition of the form

$$
A=S \operatorname{diag}\left(J_{R}, J_{C}, \bar{J}_{C}\right) S^{-1},
$$


with

$$
J_{R}=\operatorname{diag}\left(J_{1}, J_{2}, \ldots, J_{l}\right), \quad J_{C}=\operatorname{diag}\left(\hat{J}_{1}, \hat{J}_{2}, \ldots, \hat{J}_{r}\right)
$$

where $J_{k}$ is the real Jordan block corresponding to real eigenvalues $\lambda_{k}$ for $k=$ $1, \ldots, l ; \hat{J}_{k}$ is the Jordan block corresponding to complex eigenvalues $\lambda_{l+k}$, $k=1, \ldots, r$.

Theorem 8 Let the nonsingular centrohermitian matrix $A \in \mathbb{C}^{n \times n}$ have the Jordan decomposition (3.10). Assume that $s \leq l$ be the number of distinct real eigenvalues of $J_{R}$, and $t \leq r$ be the number of distinct complex conjugate eigenvalue pairs.

If $s \leq l$ or $t \leq r$, then $A$ has $2^{s+2 t}$ square roots which are functions of $A$.

If $s+t<l+r$, then $A$ has square roots which are not functions of $A$; they form $2^{l+2 r}-2^{s+2 t}$ parameterized families.

Furthermore, if $A$ has a real negative eigenvalue, then $A$ has no centrohermitian square roots which are functions of $A$;

If $A$ has no real negative eigenvalues, then $A$ has $2^{s+t}$ square roots which are polynomial in $A$ with real coefficient.

Proof. The first part is a straightforward application of Theorem 4 in [10] to centrohermitian, and the last part can be easily derived from Theorem 7 in [10] and Corollary 2.

\section{Comparison of algorithms}

In this section we will propose algorithms for computing the square roots of matrices $A$ with central symmetry, which are functions of $A$. In order to compare the computational costs of our algorithms with the standard Schur algorithm for computing a square root of a general nonsingular complex matrix, we assume that the QR Algorithm ([9, Algorithm 7.5.2]) is used. Thus, the computational costs of the Schur algorithm for computing a square root of a complex matrix $A$ are: about $25 n^{3}$ complex flops (see $[9,24]$ ) for computing Schur form $T=U^{H} A U$ where $T$ is upper triangular and has distinct eigenvalues; about $\frac{3}{2} n^{3}$ complex flops (see [9]) for computing the upper triangular square root $S=f(T)$, where $f=\lambda^{\frac{1}{2}}$ is defined on $\lambda(T)$; about $3 n^{3}$ complex flops (see [24]) for computing $X=U S U^{H}$. Therefore the total cost is about $29 \frac{1}{2} n^{3}$ complex flops.

Algorithm 1 Computes a square root of a nonsingular centrosymmetric matrix $A \in \mathbb{C}^{n \times n}$, which is a function of $A$,

where $A$ is allowed to have repeated eigenvalues of multiplicity not larger than 2 which are required to distribute in $\sigma(M)$ and $\sigma(N)$ in (2.4) respectively.

Stage 1. to compute the reduced form $P^{T} A P=\operatorname{diag}(M, N)$ in (2.4);

Stage 2. to compute the Schur decompositions $T_{M}=U_{M}^{H} M U_{M}$ and $T_{N}=$ $U_{N}^{H} N U_{N}$, respectively, where $T_{M}$ and $T_{N}$ are two upper triangular matrices;

Stage 3. to compute the upper triangular square roots $S_{M}=f\left(T_{M}\right)$ and $S_{N}=$ $f\left(T_{N}\right)$, where $T_{M}$ has $\frac{n}{2}$ distinct eigenvalues and so does $T_{N}$, and $f=\lambda^{\frac{1}{2}}$ is defined on $\lambda\left(\operatorname{diag}\left(T_{M}, T_{N}\right)\right)$; 
Stage 4. to compute $X_{M}=U_{M} S_{M} U_{M}^{H}$ and $X_{N}=U_{N} S_{N} U_{N}^{H}$;

Stage 5. to compute $X=P \quad \operatorname{diag}\left(X_{M}, X_{N}\right) P^{T}$.

The costs of Stage 1 and Stage 5 in Algorithm 1 are about $O\left(n^{2}\right)$ complex flops and are neglected. The main costs are to implement Stages 2-4. Those are about $7 \frac{3}{8} n^{3}$ complex flops. This means that our algorithm is about 4 times cheaper than the standard one.

Algorithm 2 Computes a square root of a nonsingular skew centrosymmetric matrix $A \in \mathbb{C}^{n \times n}$, which is a function of $A$, where $A$ has distinct eigenvalues.

Stage 1. to compute the reduced form $P^{T} A P=\left[\begin{array}{ll}F & E\end{array}\right]$ in (2.5);

Stage 2. compute the Schur decompositions $T=U^{H}\left(-\frac{1}{4} E F\right) U$, where $T$ is upper triangular;

Stage 3. compute the upper triangular fourth roots $S=f(T)$ (for more details, see [24]), where $T$ has $\frac{n}{2}$ distinct eigenvalues and $f=\lambda^{\frac{1}{4}}$ is defined on $\lambda(T)$;

Stage 4. solve the Sylvester equation $S \hat{Z}_{2}+\hat{Z}_{2} S=I$;

Stage 5. compute $\tilde{Z}_{2}=U^{H} \hat{Z}_{2} U$;

Stage 6. compute $Z_{2}=\tilde{Z}_{2} E$ and $Z_{3}=F \tilde{Z}_{2}$;

Stage 7. compute $Z_{4}=E^{-1} S E$;

Stage 8. form $Z$ according to (3.7);

Stage 9. compute $X=Q Z Q^{H}$.

Similarly to Algorithm 1, the dominant computational cost comes from Stages 2-7. In Stage 2, we need to compute the product $-\frac{1}{4} E F$, it takes about $\frac{1}{4} n^{3}$ complex flops by a conventional algorithm for computing matrix-matrix multiplication. The computation of the Schur decomposition of $-\frac{1}{4} E F$ requires about $\frac{25}{8} n^{3}$ complex flops. In Stage 3, to compute the upper triangular fourth roots $S=f(T)$ needs about $\frac{3}{16} n^{3}$ complex flops. The cost of Stage 4 amounts to $\frac{1}{4} n^{3}$ complex flops, see Bartels-Stewart Algorithm in [2, 9]. In Stage 5 and 6 , we need to compute $\tilde{Z}_{2}, Z_{2}$ and $Z_{3}$. Again, by conventional algorithms for computing matrix-matrix multiplication, it takes about $n^{3}$ complex flops to calculate those three matrices. Stage 7 involves a matrix-matrix multiplication and a solution of a linear system of equations with multiple right hand sides. So to do this stage needs about $\frac{7}{12} n^{3}$ complex flops. Thus the whole sum is about $5 \frac{19}{48} n^{3}$ flops, which means this structured algorithm is approximately 5.5 times cheaper than the standard one.

Algorithm 3 Computes a square root of a centrohermitian matrix A, which is a function of $A \in \mathbb{C}^{n \times n}$, where $A$ has distinct eigenvalues.

Stage 1. to compute the reduced form $R_{A}=Q^{H} A Q$ in (2.8).

Stage 2. to compute the real Schur decomposition $T=V^{T} R_{A} V$, where $T$ is a upper quasi-triangular matrix.

Stage 3. to compute $S=f(T)$ (see [10] for more details), where $T$ is upper quasi-triangular with distinct eigenvalues and $f=\lambda^{\frac{1}{2}}$ is defined on $\lambda(T)$. 
Stage 4. to compute $\tilde{S}=V S V^{T}$.

Stage 5. to compute $X=Q \tilde{S} Q^{H}$.

Again, the dominant cost in Algorithm 3 is to operate the Stage 2-4, it requires about $\frac{59}{2} n^{3}$ real flops. Note the fact that a complex addition is equivalent to 2 real additions and a complex multiplication is equivalent to 4 real multiplications and plus 2 real additions. So this structured algorithm is approximately 8 times cheaper than the standard one.

\section{Conclusions and future work}

We have exploited the reduced forms of centrosymmetric, skew-centrosymmetric and centrohermitian to study the structure of square roots of such matrices and also to design algorithms for computing those square roots. In the case of centrosymmetric matrices, the corresponding reduced forms are direct sums of matrices of half the size of the initial matrix; since the computation of a square root of a matrix of order $n$ is $O\left(n^{3}\right)$ process, computing squares roots of two matrices of order $n / 2$ involves about 4 times less arithmetic. In the case of skew-centrosymmetric matrices, the corresponding algorithm based on the reduce form involves the computation of the fourth root, the Sylvester equation and the solution of a linear equation with multiple right hands, which are of half size of the initial matrix. The new structure algorithm is approximately 5.5 times cheaper than the standard one. In the case of centrohermitian matrices, the corresponding reduce forms are real matrices of the same size of the initial matrix, structured algorithm is approximately 8 times cheaper than the standard one.

We claim that Algorithm 1 and Algorithm 3 are stable under proper conditions, based upon a simple argument similar to the ones in [3] and [10]. The stability of Algorithm 2 is more complicated because the fourth root, the Sylvester equation and the solution of a linear equation with multiple right hands are involved. We are currently exploring the stability and the accuracy of Algorithm 2 .

\section{References}

[1] A. L. Andrew, Eigenvectors of certain matrices, Linear Alg. Appl., 7 (1973), pp. 151-162.

[2] R. H. Bartels and G. W. Stewart, Solution of equation $A X+X B=C$, Comm. ACM 15 (1972), pp. 820-826.

[3] A. Björck and S. Hammarling, A Schur method for the square root of a matrix, Linear Algebra Appl., 52/53 (1983), pp. 127-140.

[4] J. A. Cadzow, Signal enhancement-a composite property mapping algorithm, IEEE Trans. Acoustics, Speech and Signal Proc., Vol. 36 (1988), pp. $49-62$. 
[5] G.W. Cross and P. Lancaster, Square roots of complex matrices, Linear and Multi-linear Algebra, 1 (1974), pp. 289-293.

[6] L. Datta and S. Morgera, On the reducibility of centrosymmetric matrices applications in engineering problems, Circuits Systems Signal Process, 8 (1989), pp. 71-96.

[7] P. I. Davis and N. J. Higham, A Parlett-Schur algorithm for computing matrix functions, SIAM J. Matrix Anal. Appl. Vol. 25 (2003), pp. 464-485.

[8] H. Faßbender and K. D. Ikramov, Computing matrix-vector products with centrosymmetric and centrohermitian matrices, Linear Algebra Appl. 364 (2003), pp. 235-241.

[9] G. H. Golub and C.F. Van Loan, Matrix Computations, Johns Hopkins University Press, Baltimore and London, 3rd edition, 1996.

[10] N. J. Higham, Computing real square roots of a real matrix, Linear Algebra Appl., 88/89 (1987), pp. 405-430.

[11] N. J. Higham, Stable iterations for the matrix square root, Numer. Algorithms, 15 (1997), pp. 227-242.

[12] N. J. Higham, Accuracy and Stability of Numerical Algorithms, SIAM, Philadelphia, PA 2002.

[13] N. J. Higham, D. S. Mackey, N. Mackey, and F. Tisseur, Function preserving matrix gorups and iterations for the matrix square root, SIAM J. Matrix Anal. Appl., Vol. 26 (2005), pp. 849-877.

[14] R. D. Hill and R. G. Betas and S. R. Waters, On centrohermitian matrices, SIAM J. Matrix Anal. Appl., Vol. 11 (1990), pp. 128-133.

[15] R. A. Horn and C. R. Johnson, Matrix Analysis, Cambridge University Press, Cambridge, UK, 1985.

[16] R. A. Horn and C. R. Johnson, Topics in Matrix Analysis, Cambridge University Press, Cambridge, UK, 1991.

[17] A. Lee, Centrohermitian and skew-centrohermitian matrices, Linear Algebra Appl., 29 (1980), pp. 205-210.

[18] H. Li and P. Stoica and J. Li, Computationally efficient maximum likelihood estimation of structured covariance matrices, IEEE Trans. Signal Proc., Vol. 47 (1999), pp. 1314-1323.

[19] Z. Y. Liu, Some properties of centrosymmetric matrices, Applied Math. Comput., 141 (2002), pp. 17-26.

[20] Z. Liu, H. Chen, and H. Cao, The computation of the principal square roots of centrosymmetric h-matrices, Appl. Math. Comp., 175 (2006), pp. 319-329. 
[21] C. D. Meyer, Matrix analysis and applied linear algebra, SIAM, Philadelphia, USA, 1st ed., 2000.

[22] B. N. Parlett, Computation of functions of triangular matrices, Memorandom, ERL-M481, Electronics Research Laboratory, College of Engeering, University of California, Berkeley, CA, 1974.

[23] B. N. Parlett, A recurrence among the elements of functions of triangular matrices, Linear Algebra Appl., 14 (1976), pp. 117-121.

[24] M. I. Smith, A Schur algorithm for computing matrix pth roots, SIAM J. Matrix Anal. Appl., Vol. 24 (2003), pp. 971-989.

[25] J. Weaver, Centrosymmetric (cross-symmetric) matrices, their basic properties, eigenvalues, and eigenvectors, Amer. Math. Monthly, 92 (1985), pp. $711-717$. 